\title{
Estimating the Expected Number of Failures to Buried Optical Fibre Cables Due to Direct Lightning Strokes by Means of a Monte Carlo Algorithm
}

\author{
Giovanni Lucca*
}

SIRTI S.p.A., Via Stamira d'Ancona 9, 20127 Milano, Italy

\begin{abstract}
This paper presents an algorithm, based on a Monte Carlo method, to assess the yearly expected number of failures to buried optical fibre cables (provided with metallic components) due to direct lightning strokes. The random parameters that have been considered in the algorithm are the lightning peak current, the distance between the cable and the point of impact of the stroke to soil and the cable failure current (i.e. the minimum value of the lightning peak current able to produce a failure on the optical fibre cable).

From this point of view, this paper proposes a new and more general approach to the problem; because, in addition to the lightning peak current, it is also based on other random parameters that should be considered for a better modelling of the phenomenon. Finally, the occurrence of the cable failures is associated to a time Poisson stochastic process.
\end{abstract}

Keywords: Monte Carlo method, risk analysis, telecommunication cables.

\section{INTRODUCTION}

It is known that optical fibre cables, provided with metallic components, (e.g. metallic sheath, service copper pairs, central strength member) are not immune from lightning threat; thus, exactly like for traditional telecommunication cables (i.e. coaxial and symmetrical pairs), it is useful to assess the yearly expected number of failures due to direct lightning strokes.

We emphasize that in this paper only direct strokes to the cable (i.e. strokes that, directly arc to the cable, so injecting the whole lightning current into the cable itself) will be considered. On the contrary, indirect strokes (i.e. strokes that do not arc to the cable so that only part of the lightning current is injected in it) will be object of a future work.

We also remind that the deleterious effects to cables associated to direct strokes, are generally much more severe than the ones associated to indirect strokes. For such a reason, assessing the number of cable failures associated to direct strokes is also of practical importance.

Actually, the assessment of the yearly expected number of failures is strictly necessary in order to quantify the risk for the cable related to keraunic activity; in fact, such information, besides other technical and economical reasons, can orient the plant owner/operator in the choice relevant to the kind of cable to be adopted.

The cable vulnerability depends, on one hand on environmental parameters as:

- $\quad$ Lightning flash density to ground (indicated by $n$ ). In some countries $\mathrm{n}$ is directly measured by lightning

*Address correspondence to this author at the SIRTI S.p.A., Via Stamira d'Ancona 9, 20127 Milano, Italy; Tel: +39 02 95885182;

Fax: +3902 95885182; E-mail: G.Lucca@sirti.it detection systems; if this is not the case, $n$ can be estimated by one of the two formulas [1]:

$$
\begin{aligned}
& n=0.04 T_{d}^{1.25} \\
& n=0.1 T_{d}
\end{aligned}
$$

where $T_{d}$ is the keraunic level defined as the number of days per year in which thunder is heard in a given location. $T_{d}$ can be generally obtained by keraunic maps.

\section{- $\quad$ Soil resistivity (indicated by $\rho$ )}

- $\quad$ Characteristics of the region (or land) through which the cable is routed: level of urbanization, orography, and significant proximity with other extended structures as power/railway lines, pipelines. These environmental characteristics are described by means of the factor $K_{e}$ [2]. Clearly, such a parameter has to be intended as a global and general average quantity that, due to its own intrinsic nature, cannot take into account of the presence in specific points, along the cable route, of certain objects (such as trees, poles) that may increase the probability of direct stroke to the cable.

On the other hand, the cable vulnerability depends also on its physical and structural characteristics that are described by a single parameter named cable failure current $I_{f}$

It is useful to remind the concept of cable failure current: it can be defined as the minimum value of lightning peak current that, arcing on the cable, is able to produce severe damages ${ }^{1}$ to the cable itself.

\footnotetext{
'e.g. breakage of one or more fibres, unacceptable increase in attenuation of the fibres, interruption in the remote power supply in the equipment (if
} 
Cable failure current $I_{f}$ is generally determined by means of a laboratory test i.e. the so called "sand box test" $[2,3]$; thus, in principle, to each kind of cable, it should be associated a value of failure current to be used as a threshold able to determine if a direct lightning stroke to the cable, can be destructive or not.

Hence, on the basis of the environmental parameters $n, \rho$ and $K_{e}$, of the cable failure current $I_{f}$, of the cable length $L$ and of the statistical lightning peak current distribution it is possible to estimate the yearly expected number of failures due to direct lightning strokes. See for example [2, 4-6].

Common feature to the above mentioned papers is that they assume the lightning peak current being the only random variable involved in the problem while the cable failure current is treated as a deterministic variable.

This last point is questionable: in fact, as well highlighted by [7], the value for $I_{f}$ obtained from to the sand box test depends, on a certain extent, on chance.

By following this reasoning, our idea is that the single value $I_{f}$ should be substituted by a range of values (e.g. the test standard deviation) that is related to the intrinsic degree of uncertainty associated to the sand box test itself; in other words, $I_{f}$ should be better considered as a random variable instead of a deterministic one.

Therefore, due to this assumption, the evaluation of the yearly expected number of failures should be done by taking into account of such a further random variable.

Another practical reason supporting this idea is that, very often, "sand box test" results are not available and one has only very few information ${ }^{2}$ concerning the parameter $I_{f}$. Thus, in practice, by grouping similar types of cable in the same family, one can adopt a range of values for $I_{f}$ instead of a single value.

Thus, in the light of these considerations, we propose a different approach that is based on a Monte Carlo method [9] that involves the three following random variables:

- $\quad$ the lightning peak current $i$

- the distance $x$ between the cable and the point of impact of the lightning stroke to soil

- the cable failure current $I_{f}$

\section{DESCRIPTION OF THE CALCULATION METHOD}

\subsection{The Monte Carlo Algorithm}

\subsubsection{Introduction}

In the case of long cables, the environmental parameters generally vary along the route, thus we have to take into account of this variability; the simplest way to manage this problem is to subdivide the cable route into a suitable number of sections each one characterized by constant

powered by metallic conductors inside the cable), destruction of the protective covering of the cable

${ }^{2}$ In paper [8] we have found some data relevant to the failure current for some kinds of cables. values for the environmental parameters $n, \rho$ and $K_{e}$. Then, at the first step, the per unit length yearly expected number of failures for each section has to be evaluated; after that, the partial results, relevant to each homogeneous section, have to be suitably added by taking into account of the section length so obtaining the result relevant to the whole cable.

\subsubsection{Per Unit Length Yearly Expected Number of Failures for a Homogenous Section}

Let us consider a section of cable $L$ where all the environmental parameters, above described, can be considered constant.

First of all, we have to define the lightning strokes collection area for the cable (see Fig. 1a); it can be represented as a rectangle having basis equal to the section length $L$ and height $2 x_{\max }$. The quantity $x_{\max }$ is related to the maximum arcing distance $d_{\max }$ to a cable for a lightning stroke to soil. See Fig. (1b).

The maximum arcing distance can be evaluated by means of formula [4]:

$d_{\max }=k(\rho) \sqrt{\rho i_{\max }}$

In (2), $k(\rho)$ is a coefficient, function of $\rho$ (expressed in $\Omega \mathrm{m})$, given by:

$k(\rho)=\left\{\begin{array}{c}0.08 \quad \rho \leq 100 \\ -3.667 \cdot 10^{-5} \rho+8.36 \cdot 10^{-2} \quad 100<\rho<1000 \\ 0.047 \quad \rho \geq 1000\end{array}\right.$

and $i_{\max }$ is the maximum value of lightning peak current (expressed in $\mathrm{kA}$ ); according to measurements, it that can be reasonably assumed equal to $250 \mathrm{kA}$.

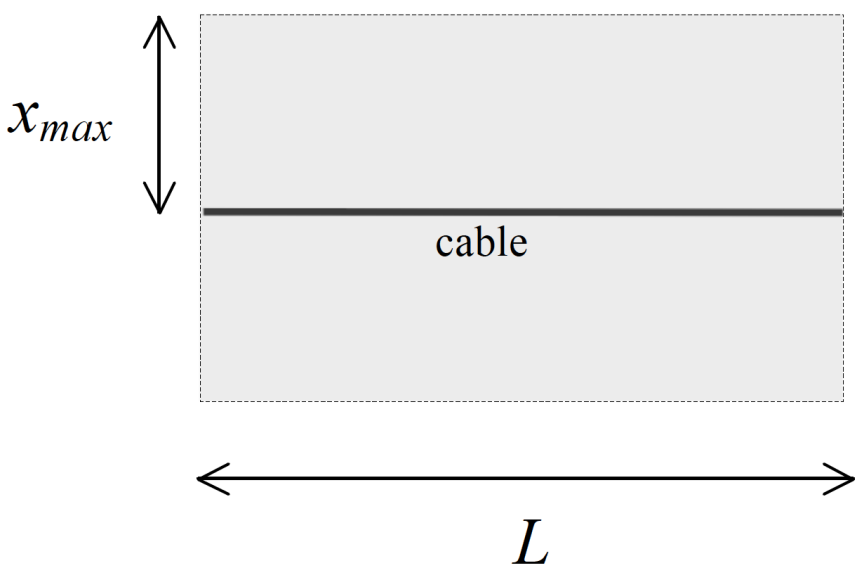

Fig. (1a). Lightning collection area for the cable.

Thus, all the lightning flashes, striking outside the rectangle shown in Fig. (1a) surely cannot arc to the cable; on the contrary, the flashes striking inside the rectangle, could arc or not to the cable. In case of arcing, the occurrence of a failure depends on the values of $i$ and $I_{f}$.

The Monte Carlo method consists (in this specific application) in generating, by means of the computer, a large number of trials where, for each trial, random values for the 
quantities listed at the end of par. 1 are chosen according to certain probabilistic laws.

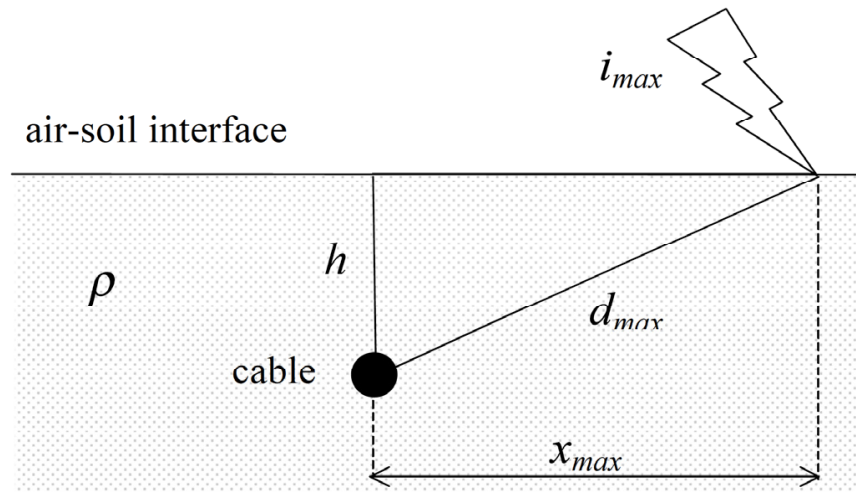

Fig. (1b). Maximum arcing distance.

By taking into account of (2), the outcome of each single trial is one among the following three:

- no arcing to the cable (see also Fig. 2) when the following condition is fulfilled:

$$
(k(\rho) \sqrt{\rho i}<h) \vee\left(x>\sqrt{k^{2}(\rho) \rho i-h^{2}}\right)
$$

- $\quad$ arcing to the cable (see also Fig. 2) when the following condition is fulfilled:

$$
(k(\rho) \sqrt{\rho i} \geq h) \wedge\left(x \leq \sqrt{k^{2}(\rho) \rho i-h^{2}}\right)
$$

- $\quad$ arcing to the cable with failure: it is a subset of the previous one described by equation (5). Its occurrence is related to the fulfilling of the following condition:

$$
(k(\rho) \sqrt{\rho i} \geq h) \wedge\left(x \leq \sqrt{k^{2}(\rho) \rho i-h^{2}} \wedge i \geq I_{f}\right)
$$

In formulas (4)-(6) the symbols $\vee$ and $\wedge$ represent the logical connectives OR and AND respectively while $i$ is the random value of the lightning peak current.

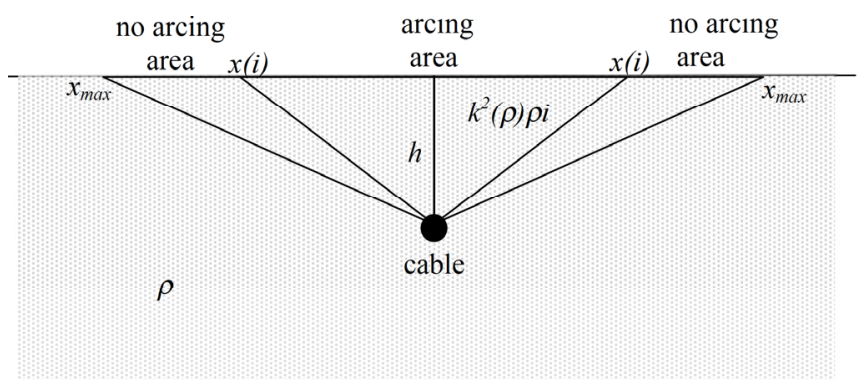

Fig. (2). Arcing and no arcing areas for the cable.

By counting each one of these three different events and by considering the ratio with the total number of trials, one obtains the probability associated to the event.

If $m_{f}$ is the number associated to the event "arc with failure" and $M$ is the number of trials, we have that the probability of failure is:

$p_{f}=\frac{m_{f}}{M}$
Therefore, the per unit length average number of yearly expected failures $N_{f}^{\prime}$ is:

$N_{f}^{\prime}=2 n K_{e} x_{\max } 10^{-3} p_{f}$

A very important point related to the Monte Carlo method is the choice of the probabilistic law associated to the random variables.

As far as the lightning peak current $i$ is concerned, according to [10], we have adopted a log-normal distribution given by the following probability density function:

$w(i)=\frac{1}{\sqrt{2 \pi} i \sigma_{\ln }} \exp \left[-\frac{1}{2}\left(\frac{\ln (i)-\ln \left(i_{m}\right)}{\sigma_{\ln }}\right)^{2}\right]$

Being $\sigma_{l n}$ the standard deviation of $\ln (i)$ and $i_{m}$ the median value of $i$. (Typical values for $i_{m}$ and $\sigma_{l n}$ are $31 \mathrm{kA}$ and 0.691 respectively).

For the lateral distance $x$ between striking point and cable, it is reasonable to assume a uniform distribution in the interval $\left[0, x_{\max }\right]$

In relation to the cable failure current $I_{f}$, one should start from the result of the "sand box test" (if available) $I_{s b t}$ and to consider the random variable $I_{f}$ uniformly distributed inside the range $\left[I_{s b t}-\Delta, I_{s b t}+\Delta\right] . \Delta$ can be $10 \%-20 \%$ of the value of $I_{s b t}$. Also in this case, the assumption of the uniform distribution seems reasonable and, in many cases, may be justified by the lack of experimental data.

\subsubsection{Yearly Expected Number of Failures for the Whole Cable}

If the whole cable is composed by a number $Q$ of homogeneous sections and the generic k-th one is characterized by the values $N_{f k}^{\prime}$ and length $L_{k}$, the yearly expected number of failures is given by:

$N_{f}=\sum_{k=1}^{Q} N_{f k}^{\prime} L_{k}$

The quantities $N_{f k}^{\prime} \cdot L_{k}$ represent the partial results relevant to each section; they give an indication about the more exposed regions, along the route, to lightning strokes. Therefore, if needed, some protective countermeasures can be adopted (e.g. the use of one or two shield wires) only in those particular sections where the value $N_{f k}=N_{f k}^{\prime} \cdot L_{k}$ is deemed too high.

\subsection{Cable Failures Occurrence as a Poisson Process}

The knowledge of $N_{f}$ is certainly an useful quantity in order to estimate the risk represented by direct lightning strokes; nevertheless, we can get a more complete information if we also know the probability of having a certain number $j$ of failures in a generic time interval $[0, t]$. That means to be able to describe our problem by means of a time stochastic process where the random events are represented by the cable failures due to direct lightning strokes.

The stochastic process which can properly describe a typical counting problem of "rare events", as the one we are 
dealing with, is the Poisson process [11]. According to it, the probability $p(j, t)$ of having $j$ failures in the time interval $[0$, $t]$ is given by:

$p(j, t)=\frac{\left(N_{f} t\right)^{j}}{j !} e^{-N_{f} t}$

From (11) we can obtain the probability $P(j, t)$ of having a number of failures greater than $j$ in the time interval $[0, t]$ i.e.:

$P(j, t)=1-\sum_{k=0}^{j} \frac{\left(N_{f} t\right)^{k}}{k !} e^{-N_{f} t}$

Note that the quantity $N_{f} t$ represents the mean value of the random variable: "number of failures in the time interval $[0, t] "$.

\section{EXAMPLES OF APPLICATION}

The first example of application deals with three different optical fibre cables each one characterized by its own range of failure current $I_{f}$. The cables are buried at a depth of $1 \mathrm{~m}$ in a region characterized by $n=1$ strokes/(year $\mathrm{km}^{2}$ ), and environmental factor $K_{e}=1$; we have evaluated the per unit length yearly expected number of failures versus the soil resistivity. The number $M$ of trials processed for the Monte Carlo simulation is 50000 .

In Fig. (3), the plots of the per unit length yearly expected number of failures $N_{f}$ versus the soil resistivity, for different ranges of the failure current $I_{f}$, are shown.

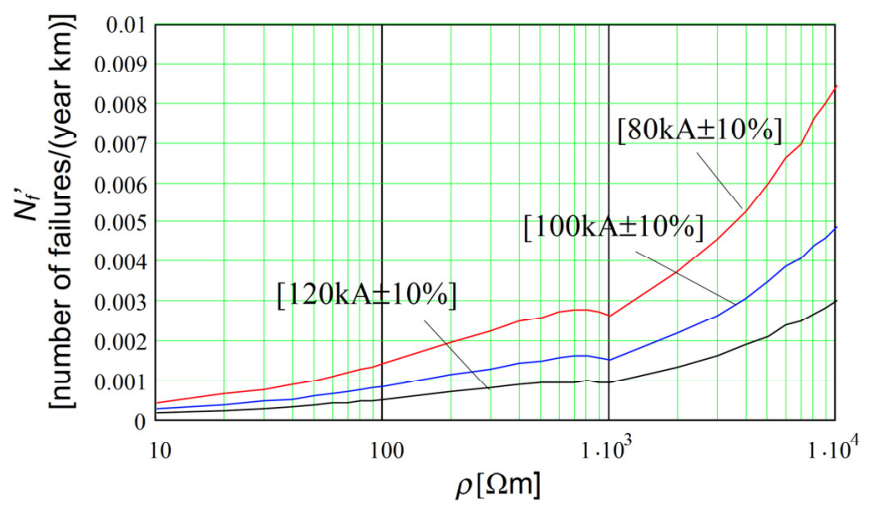

Fig. (3). Per unit length yearly expected number of failures versus soil resistivity for different ranges of failure current.

Fig. (3) confirms that the soil resistivity is an important parameter that influences the level of risk for the cable; at the same time, we can see that the weaker is the cable, (i.e. having low values for the failure current range), the larger is the number of yearly failures to be expected.
We also present an application of formula (12), by considering a cable, $100 \mathrm{~km}$ long, placed in a homogeneous region (characterized by parameters $K_{e}=1$ and $n=1$ strokes/(year $\left.\left.\cdot \mathrm{km}^{2}\right)\right)$ and a time period of 25 years that can be considered the typical life of an optical fibre cable; Fig. (4) represents the percent probability of having a number of failures greater than the integer number shown in abscissa. The plots are corresponding to two different soil resistivities $(100 \Omega \mathrm{m}$ and $1000 \Omega \mathrm{m})$ while the failure current range is [100kA $\pm 10 \%]$.

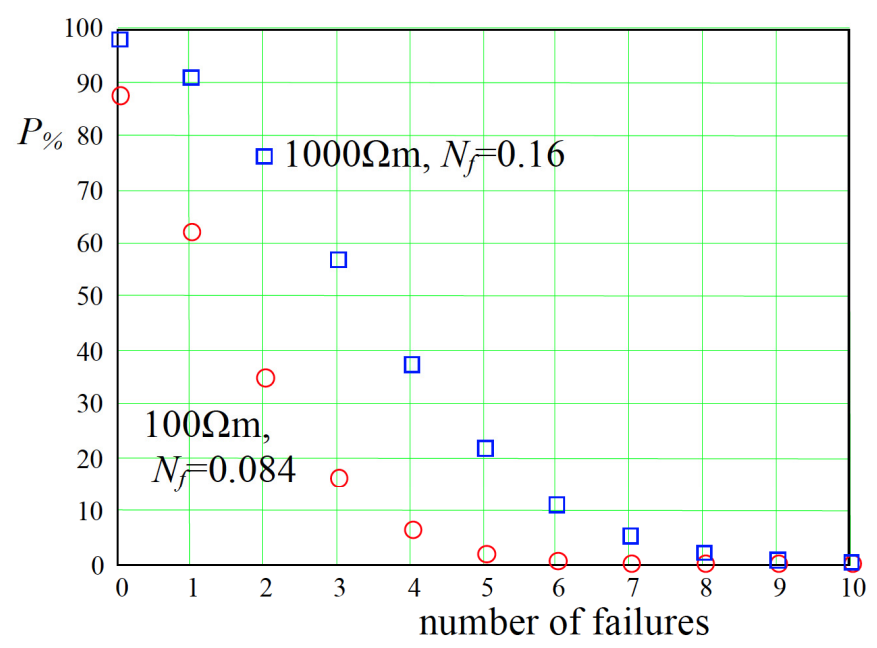

Fig. (4). Percent probability of having a number of failures greater than the number shown in abscissa in a period of 25 years.

The next example, deals with a cable $300 \mathrm{~km}$ long that crosses four regions having different characteristics reported in Table 1.

In the last column, we have reported the results of the Monte Carlo calculations relevant to each region.

By using them and by applying formula (10), we have that the total number of yearly expected failures on the cable is 0.864 failures/year.

By comparing the partial results and by looking at Fig. (5), we can see that most of the failures are concentrated in region 2 which can be considered the most exposed one.

Thus, in order to reduce the number of yearly expected failures in region 2 , an effective mitigation countermeasure is given by the use of one or two shield wires installed some tens of centimetres above the cable itself; the shielding mechanism is sketched in Fig. (6).

We remind that the shield wire(s) effect is to divert part of the lightning current $i$ injected on the cable; thus, if a

Table 1. Characteristics of the Regions Crossed by the Cable and Results of Calculations

\begin{tabular}{|c|c|c|c|c|c|}
\hline Region (Section) & Length $[\mathbf{k m}]$ & $\rho[\mathbf{\Omega m}]$ & $K_{e}$ & $n\left[\right.$ Strokes/(Year km $\left.\left.{ }^{2}\right)\right]$ & $N_{f}[$ Failures/Year] \\
\hline \hline 1 & 100 & 100 & 1 & 1 & 0.085 \\
\hline 2 & 50 & 2000 & 1 & 4 & 0.427 \\
\hline 3 & 100 & 1000 & 1 & 2 & 0.313 \\
\hline 4 & 50 & 500 & 0.5 & 1 & 0.039 \\
\hline
\end{tabular}




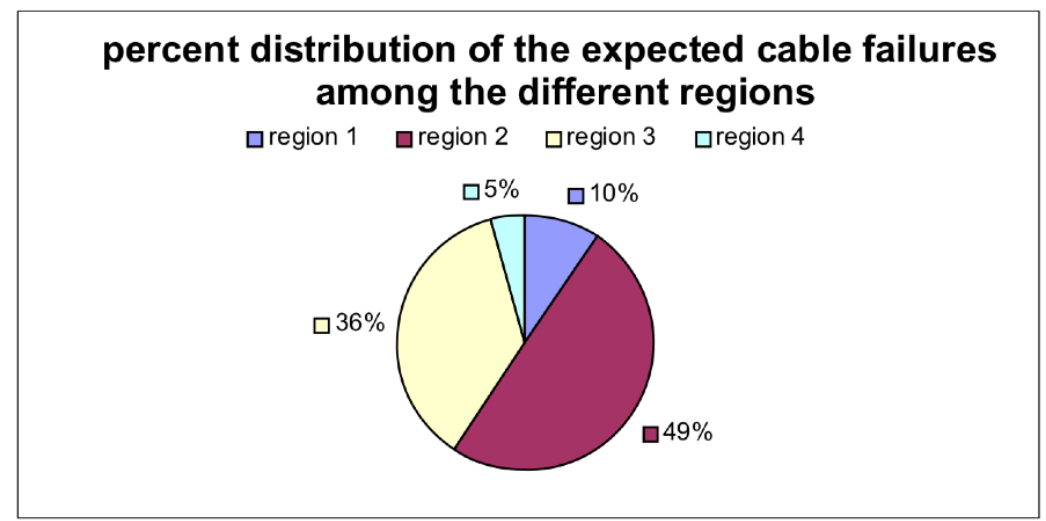

Fig. (5). Percent distribution of the yearly expected number of failures among the different regions crossed by the cable.

shield wire is characterized by a shielding factor $\eta(0<\eta<1)$, the actual current injected on the cable is $\eta \mathrm{i}$. Formulas and tables for evaluating the shielding factor $\eta$, for different geometrical disposition of the shield wire(s) can found in [2].

According to [2], the shielding factor $\eta$ associated to one shield wire is about 0.5-0.6 while, in the case of two shield wires is about 0.35 . So, by installing shield wires only in region 2, we obtain the results shown in Table 2.

The reduction in the number of yearly failures is very significant if related to the only region 2, but is less noticeable if related to the whole cable.

In Fig. (7), we have compared the probability of having a number of failures greater than the integer shown in abscissa in a period of 25 years by considering the cable with and without shield wires.
Table 2. Reduction of Failures Due to the Presence of Shield Wire(s); Shield Wire(s) Installed Only in Region 2

\begin{tabular}{|c|c|c|c|}
\hline & $\eta$ & $\boldsymbol{N}_{f 2}$ [Failures/Year] & $\boldsymbol{N}_{\boldsymbol{f}}$ [failures/year] \\
\hline \hline no shield wire & 1 & 0.427 & 0.864 \\
\hline 1 shield wire & 0.55 & 0.066 & 0.503 \\
\hline 2 shield wires & 0.35 & 0.01 & 0.447 \\
\hline
\end{tabular}

From the plots, it appears that the difference in the results between the case of one and two shield wires is quite small, at least, in this case.

On the contrary, if the shield wire(s) is (are) installed along the whole cable route, we obtain the result reported in Table 3 and in Fig. (8).

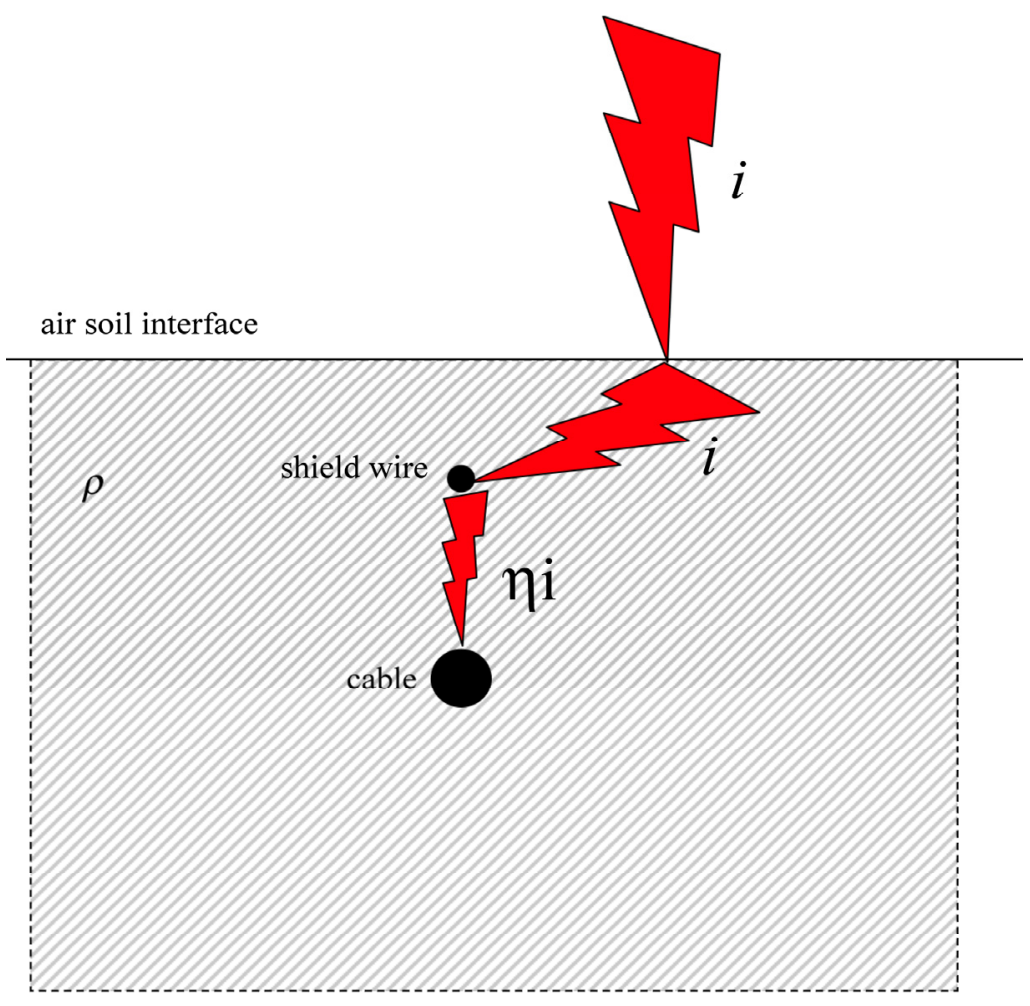

Fig. (6). Shielding mechanism for direct strokes. 


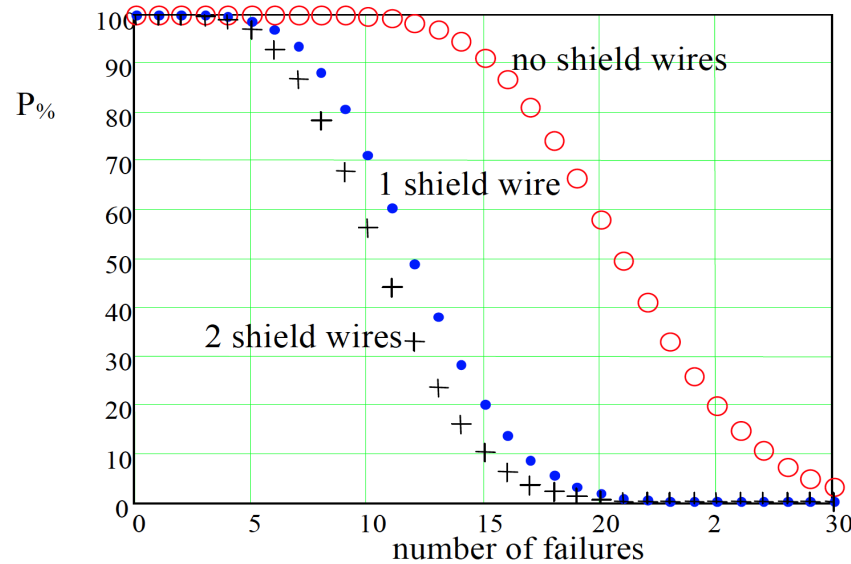

Fig. (7). Percent probability of having a number of failures greater than the number shown in abscissa in a period of 25 years. Shield wire(s) installed only in region 2.

Table 3. Reduction of Failures Due to the Presence of Shield Wire(s); Shield Wire(s) Installed Along the Whole Cable Route

\begin{tabular}{|c|c|c|}
\hline & $\boldsymbol{\eta}$ & $\boldsymbol{N}_{f}[$ Failures/Year] \\
\hline \hline no shield wire & 1 & 0.864 \\
\hline 1 shield wire & 0.55 & 0.129 \\
\hline 2 shield wires & 0.35 & 0.02 \\
\hline
\end{tabular}

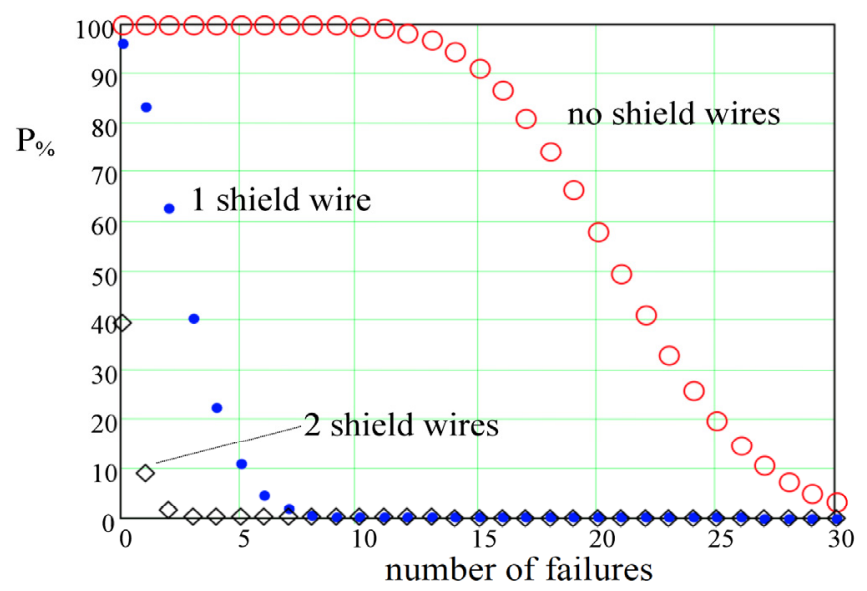

Fig. (8). Percent probability of having a number of failures greater than the number shown in abscissa in a period of 25 years. Shield wire(s) installed along the whole cable route.

The use of shield wire(s) along the whole cable route strongly reduces the number of yearly expected failures and noticeable difference exists, in this case, between the results obtained by using one or two shield wire.

\section{CONCLUSIONS}

The paper presents an algorithm, based on a Monte Carlo method, to estimate the number of yearly expected failures relevant to an optical fibre cable due to direct lightning strokes. The method allows taking into account of the intrinsic random nature, not only of the lightning peak current, but also of the point of impact to soil of the lightning stroke and of the cable failure current; the latter one being a parameter qualifying the cable itself. From this point of view, the paper proposes a new and more general approach to the problem.

There is also an actual advantage in the approach presented because, in order to apply it, we do not need an exact value for the cable failure current, but a range of values. In fact, data relevant to such a parameter are often lacking and/or difficult to collect if referred to a specific cable; on the contrary, it is easier and more practical to associate to different cables, but having common characteristics, a suitable interval of values.

\section{ACKNOWLEDGEMENT}

Declared none.

\section{CONFLICT OF INTEREST}

Declared none.

\section{REFERENCES}

[1] ITU T. Recommendation K.47 "Protection of telecommunication lines using metallic conductors against direct lightning discharges". ITU: Geneva 2008.

[2] ITUT. Recommendation K.25 "Protection of optical fibre cables". ITU: Geneva 2000.

[3] TIA/EIA Standard FOTP-181 Lightning damage susceptibility test for fiber optic cables with metallic components. American National Standard; ANSI/TIA/EIA 1992; pp. 455-181

[4] Sunde ED. Earth conduction effects in transmission systems. New York, Van Nostrand Company Inc. 1949; p .373.

[5] CCITT. The protection of telecommunication lines and equipment against lightning discharges. Geneva, ITU 1995.

[6] Lucca G. Risk assessment and lightning protection system for buried telecommunication cables Proc. of $23^{\text {rd }}$ International Conference on Lightning Protection, Firenze: Italy, 1996.

[7] Stingl U, Greiner W. Investigation of the lightning damage susceptibility of fiber optic cable. Proc $53^{\text {rd }}$ International Wire and Cable Symposium 2004: Philadelphia, USA.

[8] Clerico G, Solbiati GL. Lightning discharges and electromagnetic induction on optical fibre plants. Proc $13^{\text {th }}$ European Fibre Optic Communications EFOC 1995: Brighton, UK.

[9] M. Sobol, "The Monte Carlo Method", 2nd Edition, MIR Publishers, Moscow, 1984

[10] Chowdhuri P. Electromagnetic transients in power systems. Research Studies Press LTD, Taunton, Somerset, England 1996.

[11] Lucca G. A Poisson process to estimate the probability of failures to buried telecommunication cables due to lightning strokes to ground. Proc. of $24^{\text {th }}$ International Conference on Lightning Protection 1998: Birmingham, UK. 